Article published in Physica Scripta T128 (2007) 35

\title{
Long-term erosion and deposition studies of the main graphite limiter in TEXTOR
}

\author{
A. Kreter ${ }^{\mathrm{a}}$, A. Kirschner ${ }^{\mathrm{a}}$, P. Wienhold ${ }^{\mathrm{a}}$, J. Likonen ${ }^{\mathrm{b}}$, M. Mayer ${ }^{\mathrm{c}}$, V. Philipps ${ }^{\mathrm{a}}$, U. Samm ${ }^{\mathrm{a}}$, \\ G. Sergienko ${ }^{a}$ and TEXTOR team ${ }^{\mathrm{a}}$ \\ ${ }^{a}$ Institut für Plasmaphysik, Forschungszentrum Jülich, Association EURATOM-FZJ, Trilateral Euregio Cluster, \\ Germany \\ ${ }^{b}$ Association EURATOM-TEKES, VTT , PO Box 1000, 02044 VTT, Espoo, Finland \\ ${ }^{c}$ Max-Planck-Institut für Plasmaphysik, EURATOM Association, 85748 Garching, Germany
}

PACS: 52.40.Hf, 52.55.Fa, 82.80.Ms

\section{Abstract}

Erosion and deposition behaviour of the TEXTOR main toroidal belt limiter ALT-II has been studied for two generations of limiter tiles with different surface shapes. Two specially prepared tiles with marker holes were subsequently exposed during experimental campaigns with a total plasma duration of $>7000 \mathrm{~s}$ and a surface averaged background ion fluence exceeding $2 \cdot 10^{25} \mathrm{~m}^{-2}$. The tile surface relief was measured by optical profilometry and SIMS before and after the exposure. The highest surface erosion of $>10 \mu \mathrm{m}$ on both tiles was observed in regions with an angle of incidence of the magnetic field $\alpha$ of $>\sim 1^{\circ}$, whereas in the regions with $\alpha \sim 0.1^{\circ}$ both net-erosion and net-deposition were observed depending on the tile shaping. The marker holes were always deposition dominated. Modelling by the ERO code explains qualitatively the surface erosion and deposition behaviour in terms of background flux dilution for shallow angles of incidence.

\section{Introduction}

TEXTOR is a medium size tokamak (major radius $1.75 \mathrm{~m}$, minor radius $0.46 \mathrm{~cm}$ ) with a circular poloidal plasma cross-section [1]. The position of the last closed flux surface (LCFS) is defined by the toroidal belt limiter ALT-II (Advanced Limiter Test II) [2]. ALT-II consists of 8 blades, each carrying 28 tiles ordered in two rows, with a total surface area of $3.4 \mathrm{~m}^{2}$. The tiles are produced of fine-grain isotropic graphite. Two different shapes of the plasma-wetted tile surface have been employed (Fig. 1 (a,b)). The original tiles [3] first installed in 1987 
have a shape with a tangential geometry (angle of incidence of the magnetic field $\alpha$ of $\sim 0.1^{\circ}$ ) for the central $2 / 3$ of the poloidal range of the blade. The edges curve away with $\alpha$ increasing by several degrees deeper in the scrape-off layer (SOL). This shaping caused non-uniform heat flux distributions $[3,4]$ with higher fluxes towards the steep edge of the tile. The tiles are flat toroidally, leading to a toroidal non-uniformity of the power load.

The shape of the refined tiles was designed to extend the region with $\alpha<1^{\circ}$ to $10 \mathrm{~mm}$ in the SOL, resulting in a spread of the heat flux to a larger effective area [5]. Toroidally the tiles are shaped according to the toroidal curvature of the LCFS. The original tiles were replaced by the refined tiles in 1995.

During the experimental campaign in 2000 a specially prepared tile was installed to investigate the long-term erosion and deposition behaviour of the ALT-II limiter with the refined shape [6,7]. Its surface had a number of $\sim 0.3 \mathrm{~mm}$ deep drill holes of $5 \mathrm{~mm}$ diameter. The bottoms of the holes were protected from erosion and used as reference points for surface relief measurements by optical profilometry and SIMS. After exposure for 7625 plasma seconds a maximum deposition of about $40 \mu \mathrm{m}$ was observed in the central region (about 1/3 of the poloidal range) where $\alpha \sim 0.1^{\circ}$, whereas the area with a steeper angle of incidence of $\sim 3^{\circ}$ was subject to net-erosion of up to $28 \mu \mathrm{m}$.

In the campaign 2003-2004 the tiles of the original shape were re-installed. This opportunity was taken to repeat the experiment with a similarly prepared tile of the original shape and to compare the erosion and deposition behaviour of the ALT-II tiles of different shapes.

\section{Experiment and analysis}

\subsection{Long-term exposure of the special ALT-II tile}

A tile of the original shape was specially prepared to quantify its erosion and deposition behaviour. Holes of a depth of $0.25-0.35 \mathrm{~mm}$ ordered in 3 toroidal columns and 10 poloidal rows were drilled in the hand-polished tile surface to serve as erosion-protected markers. The 
surface relief was pre-characterised by optical profilometry. To exclude a possible influence of the toroidal asymmetry, e.g. field ripple, the tile was installed at the same position in TEXTOR (ALT-II blade 5, tile 20) as the previously investigated tile of the refined shape. Fig. 1 (c) shows a photograph of the special tile after the exposure in a complete TEXTOR experimental campaign with a total plasma duration of 7085 s. The visual inspection showed that the tile is qualitatively representative for the neighbouring tiles.

The area averaged background fluxes were routinely determined by the integration of the $\mathrm{H}_{\alpha}$ light over the entire poloidal extension of the limiter blade.

Table I summarises the main properties of the plasma exposure of both tiles. The campaigns were similar in their duration and integral plasma parameters. It allows a direct comparison of the tile performances. It has to be mentioned, that the campaign with the original tile was performed after the installation of the Dynamic Ergodic Divertor (DED) in TEXTOR. The DED operation favours a plasma position shift by several $\mathrm{cm}$ towards the DED target at the high field side (HFS) of the torus. It results in a substantially different particle flux distribution at the ALT-II limiter. About one quarter of the campaign programmes were performed with a HFS shift.

Integral surface erosion and deposition for the tile, which are the topic of the next sub-section, are also given in Table I.

\subsection{Post-mortem analysis}

The experiment confirmed the earlier finding [7], that a marker hole recess of only several tenths of a mm turns an erosion dominated area in a deposition dominated one. For the shallow angles of incidence discussed here most of the impinging particles are scrapped-off at the border of the hole and do not reach its bottom. Only hydrocarbon molecules or radicals can contribute to deposition on the bottom of the hole due to their large gyroradii of several mm. Therefore, the deposition rates in the holes can be considered as a measure for the chemical erosion in the vicinity. The thickness of deposition in the marker holes was 
measured by the SIMS sputter depth profiling. Fig. 2 (a) shows the layer thicknesses for the holes of the column II for both tiles. Columns I and III have similar distributions of deposition in the holes.

Deposition on the bottom of the holes stands in competition with erosion by charge-exchange (CX) neutrals. Previous findings in TEXTOR [8] showed, that erosion of the shadowed limiter surface by CX neutrals has typical rates of $0.1 \mathrm{~nm} / \mathrm{s}$. This is about one order of magnitude less than the deposition rates observed in the holes. Therefore, the effect was not considered in the calculations of the surface profile evaluation.

The thicknesses of the deposited layers in the marker holes were used as offsets to compare the optical profilometry measurements before and after the exposure. In Fig. 2 (b) the evolution of the surface profile along the central column is plotted. Fig. 3 shows a 2dimensional reconstruction of the erosion and deposition pattern of the entire tile.

An extensive error analysis of this method of the surface profile measurements was done in [7]. Applying this analysis for the original tile, the estimated errors in the determined erosion and deposition pattern vary from $\pm 2 \mu \mathrm{m}$ in the tangential part of the tile to $\pm 5 \mu \mathrm{m}$ for the drill hole rows $1-2$, where the hole bottoms are strongly tilted with respect to the tile surface ( $c f$. [7]).

\section{Modelling and discussion}

\subsection{Modelling by the ERO code}

The erosion and deposition behaviour of the tile with the refined shape was simulated by the ERO code [9]. The input data for the calculations were taken according to the findings from the studies of carbon transport in TEXTOR [10]: a chemical erosion yield of graphite of $2 \%$, an enhanced chemical erosion yield of freshly deposited layers of 8\%, zero effective sticking of hydrocarbons, sticking of atoms according to the TRIM code and a fraction of carbon in the background flux of 3\% were assumed as input parameters. Campaign averaged edge plasma parameters were taken: $T_{e}^{L C F S}=54 \mathrm{eV}$ with an exponential decay length in the SOL of 
$40 \mathrm{~mm}, T_{i}^{L C F S}=81 \mathrm{eV}$ with the same decay length and $n_{e}{ }^{L C F S}=1.9 \cdot 10^{18} \mathrm{~m}^{-3}$ with a decay length of $22 \mathrm{~mm}$. An exposure duration of $5 \mathrm{~s}$ (10 calculation steps of $0.5 \mathrm{~s}$ each) was simulated, so that steady-state in terms of erosion and deposition rates was reached. The effect of sheath modification at the shallow angles of incidence was not considered in these calculations.

The calculations reproduced qualitatively the evolution of the surface profile in poloidal direction (Fig. 4) with an erosion dominated area at the lower edge of the tile, which transforms to a deposition dominated area towards the upper edge (cf. Fig. 2 (b)). Note, that the upper edge of the investigated tile corresponds to the central poloidal zone of the ALT-II blade, which defines the LCFS (Fig. 1).

Two effects are likely responsible for this behaviour of the surface profile. The first one is the dilution of the background plasma flux, which is responsible for erosion, corresponding to the angle of magnetic field incidence $\alpha$. It leads to highest erosion rates at the lower edge of the tile with $\alpha$ of up to $2.5^{\circ}$ and reduced erosion towards the upper edge where $\alpha \sim 0.1^{\circ}$. Secondly, the particles eroded from the tile surface are partly ionised in the SOL and transported by the SOL flow directed towards the upper edge of the tile. The calculations showed, that about $90 \%$ of the physically sputtered carbon atoms leave the SOL before they are ionised and, therefore, do not contribute to deposition. Chemically eroded carbon, on the contrary, is locally re-deposited with a probability of about $90 \%$. The step-by-step mechanism of chemical erosion, the SOL migration and subsequent re-deposition is responsible for the effective carbon transport towards the poloidal blade centre. Here carbon remains because of reduced erosion.

Further parametric studies with the ERO code for both shapes of the ALT-II limiter are ongoing and will be a subject of a later publication.

\subsection{Comparison of erosion and deposition for differently shaped tiles}


The poloidal distribution of surface erosion and deposition is qualitatively similar for the original and refined tiles (Fig. 2 (b)). Erosion with highest rates at the lower edges dominates about $2 / 3$ of the poloidal extension of the tiles. The upper parts of the tiles are deposition dominated. However, there are substantial quantitative differences between both tiles in the surface profile evolution. In the case of the original tile, the zone with the highest erosion rates corresponds to the lower part of the tile, where the surface profile curves away from the LCFS (Fig. 1 (a)). In the part of the tile tangential to the LCFS, the erosion and deposition rates are low. Unlike the refined tile, no thick deposited layers in the upper part of the original tile were observed.

The reason for the different behaviour is the difference in the tile geometry. The area with the surface non-tangential to the LCFS of the original tile (from the lower edge to the drill hole row 3) is comparable to the entire poloidal range of the refined tile. Indeed, the surface erosion rates in this zone of the original tile are similar to the rates in the erosion dominated area of the refined tile. Moreover, the deposition distribution in the drill holes for rows $1-3$ of the original tile with an increase towards the LCFS is similar to the situation for the entire poloidal range of the refined tile. Carbon is driven across the surface by the step-by-step transport towards the LCFS described in section 3.1, with deposition in the holes acting as an indicator for the accumulated hydrocarbon flux.

In analogy to the massive carbon deposition in the areas closest to the LCFS observed at the refined tile, one would expect an accordingly high deposition at the corresponding zone of the original tile (drill hole row 4). However, no massive deposition was observed here. On the contrary, this area is erosion dominated. It can possibly be explained by the quite frequent shifts of the plasma position towards the HFS as mentioned in section 2.1. During these shifts the plasma 'sits' on the convexly shaped zone of the lower ALT-II tiles (corresponding to drill hole rows $1-4)$, causing higher background fluxes and, correspondingly, higher erosion in the area, where geometry is tangential in case of the central plasma position (drill hole rows 
$4-10$ ). However, the last $\approx 800 \mathrm{~s}$ of the plasma operation of the campaign were carried out with the central plasma position. This might explain the slight deposition in the entire tangential region (rows 4 -10) observed visually after the exposure (Fig. 1 (c)).

Unlike the refined tile, a noticeable local toroidal asymmetry was found in erosion of the original tile. The amounts of eroded material indicated in the lower part of the Fig. 3 are on the right-hand side by a factor of $\approx 1.5$ higher than on the left-hand side. It is a result of the toroidally flat profile of the original tile, which causes a toroidal asymmetry in the angle of incidence $[3,4]$ and a corresponding asymmetry in the particle and heat fluxes.

The integral erosion rate (Table I) is for the original tile by a factor of 2 lower than for the refined tile. Averaged background fluxes to the limiter of $3.6 \cdot 10^{21} \mathrm{~m}^{-2} \mathrm{~s}^{-1}$ for the original tile and $3.0 \cdot 10^{21} \mathrm{~m}^{-2} \mathrm{~s}^{-1}$ for the refined one do not differ much. The eroded amounts of carbon correspond to erosion yields of $2.5 \%$ and $4.1 \%$ for the original and refined tiles, respectively. The lower value for the original tile might be due to the smaller non-tangential region, which reduces the effective interaction area to about $1 / 3$ of the total area. This causes an increase of the particle flux to $>10^{22} \mathrm{~m}^{-2} \mathrm{~s}^{-1}$, resulting in lower yields of chemical erosion [11], which has a significant contribution to the total erosion rates.

\section{Conclusions}

The erosion and deposition behaviour was investigated for two generations of the ALT-II limiter in long-term experiments using specially prepared tiles. Both limiters were exposed in TEXTOR campaigns to similar integrated plasma parameters allowing a direct comparison of the surface profile evolution.

The differences in the tile geometry led to different erosion and deposition patterns on both tiles. In case of the original tile, the plasma-limiter interaction was primarily focused to the non-tangential zone, whereas for the refined tile the particle and heat fluxes were distributed more uniformly. Lower effective interaction area of the original tile led to reduced erosion rates compared to the refined tile. However, the tile with the original shape is more sensitive 
to the shifts of the plasma position, resulting in a higher power load of the convexly shaped limiter zones. More uniform shape of the refined tile is certainly more tolerable concerning possible errors in the plasma positioning.

The importance of the tile shaping is underlined by the fact, that the toroidal shaping of the refined tile reduced significantly the toroidal asymmetry of the erosion pattern observed for the original tile.

Marker holes selectively collecting hydrocarbon molecules in their vicinity showed that chemical erosion has the highest rates at the edges of the non-tangential limiter regions closest to the LCFS.

Modelling with the ERO code was able to explain qualitatively the erosion and deposition behaviour of the refined limiter. The background flux, which is responsible for carbon erosion, is diluted corresponding to the angle of incidence of the magnetic field. In combination with the step-by-step migration of the chemically eroded carbon it results in a net transport to regions of shallow angles near the LCFS.

\section{References}

[1] Samm, U., Fusion Sci. Technol. 47, 73 (2005).

[2] Finken, K. H, Reiter, D., Denner, T., Dippel, K. H., Hobirk, J., Mank, G., Kever, H., Wolf, G. H., Noda, N., Miyahara, A., Shoji, T., Sato, K. N., Akaishi, K., Boedo, J. A., Brooks, J. N., Conn, R. W., Corbett, W. J., Doerner, R. P., Goebel, D., Gray, D. S., Hillis, D. L., Hogan, J., McGrath, R. T., Matsunaga, M., Moyer, R., Nygren, R. E., and Watkins, J., Fusion Sci. Technol. 47, 126 (2005).

[3] McGrath, R. T., Fusion Engrg. Des. 13, 267 (1990)

[4] Finken, K. H., Baeck, W. Y., Dippel, K. H., Boedo, J. A., Gray, D. S., Mank, G., J. Nucl. Mater. 196-198, 220 (1992)

[5] Denner, T., Finken, K. H., Mank, G., Noda, N., Nucl. Fusion 39, 83 (1999) 
[6] Wienhold, P., Philipps, V., Kirschner, A., Huber, A., von Seggern, J., Esser, H. G., Hildebrandt, D., Mayer, M., Rubel, M., Schneider, W., J. Nucl. Mater 313-316, 311 (2003)

[7] Mayer, M, Wienhold, P., Hildebrandt, D., Schneider, W., J. Nucl. Mater 313-316, 377 (2003)

[8] Wienhold, P., Rubel, M., Mayer, M., Hildebrandt, D., Schneider, W., Kirschner, A., Physica Scripta T94, 141 (2001)

[9] Kirschner, A., Philipps, V., Winter, J., Kögler, U., Nucl. Fusion 40, 989 (2000)

[10] Kirschner, A., Wienhold, P., Philipps, V., Coad, J. P., Huber, A., Samm, U., JET EFDA contributors, J. Nucl. Mater 328, 62 (2004)

[11] Roth, J., Preuss, R., Bohmeyer, W., Brezinsek, S., Cambe, A., Casarotto, E., Doerner, R., Gauthier, E., Federici, G., Higashijima, S., Hogan, J., Kallenbach, A., Kirschner, A., Kubo, H., Layet, J. M., Nakano, T., Philipps, V., Pospieszczyk, A., Pugno, R., Ruggieri, R., Schweer, B., Sergienko, G., and Stamp, M., Nucl. Fusion 44, L21 (2004) 
Table I. Summary of the long-term experiments with the ALT-II special tiles. Erosion and deposition are determined for the erosion or deposition dominated parts of the limiters, respectively.

\begin{tabular}{|c|c|c|c|c|c|c|c|}
\hline $\begin{array}{l}\text { Experiment } \\
\text { (year of }\end{array}$ & $\begin{array}{l}\text { Duration of } \\
\text { exposure [s] }\end{array}$ & $\begin{array}{l}\text { Background } \\
\text { ion fluence }\end{array}$ & $\begin{array}{l}\text { Averaged } \\
\text { background }\end{array}$ & $\begin{array}{l}\text { Carbon } \\
\text { erosion of }\end{array}$ & $\begin{array}{l}\text { Carbon re- } \\
\text { deposition }\end{array}$ & $\begin{array}{l}\text { Erosion rate } \\
\text { for entire }\end{array}$ & $\begin{array}{l}\text { Re-deposition } \\
\text { rate for entire }\end{array}$ \\
\hline \multirow[t]{2}{*}{ exposure) } & & {$\left[\mathrm{m}^{-2}\right]$} & ion flux & special tile & on special & limiter (224 & limiter (224 \\
\hline & & & {$\left[\mathrm{m}^{-2} \mathrm{~s}^{-1}\right]$} & [mg] & tile [mg] & tiles) $[\mathrm{g} / \mathrm{h}]$ & tiles) $[\mathrm{g} / \mathrm{h}]$ \\
\hline Original tile & 7085 & $2.5 \cdot 10^{25}$ & $3.6 \cdot 10^{21}$ & 94 & 9 & 11 & 1.0 \\
\hline \multicolumn{8}{|l|}{ (2003-2004) } \\
\hline Refined tile & 7625 & $2.4 \cdot 10^{25}$ & $3.0 \cdot 10^{21}$ & 210 & 96 & 22 & 10 \\
\hline (2000) & & & & & & & \\
\hline
\end{tabular}




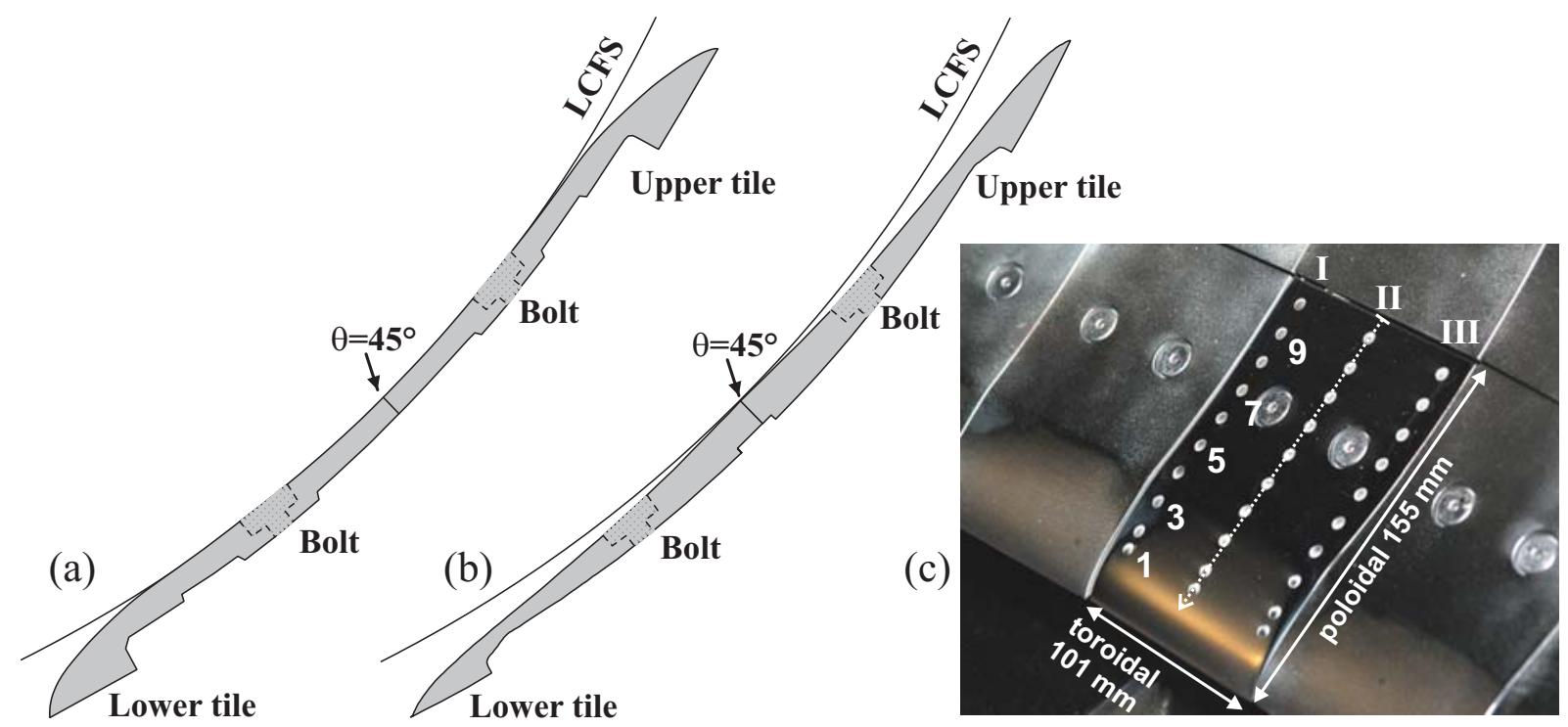

Fig. 1. Geometry of the ALT-II limiter: Poloidal cross-section of the ALT-II blade with (a) original and (b) refined shape; (c) View of the special tile with the original shape after the exposure. The dimensions of the tile and the positions of the marker holes are indicated. The dotted line represents the position and the direction of the $\mathrm{X}$ axis in Fig. 2. 

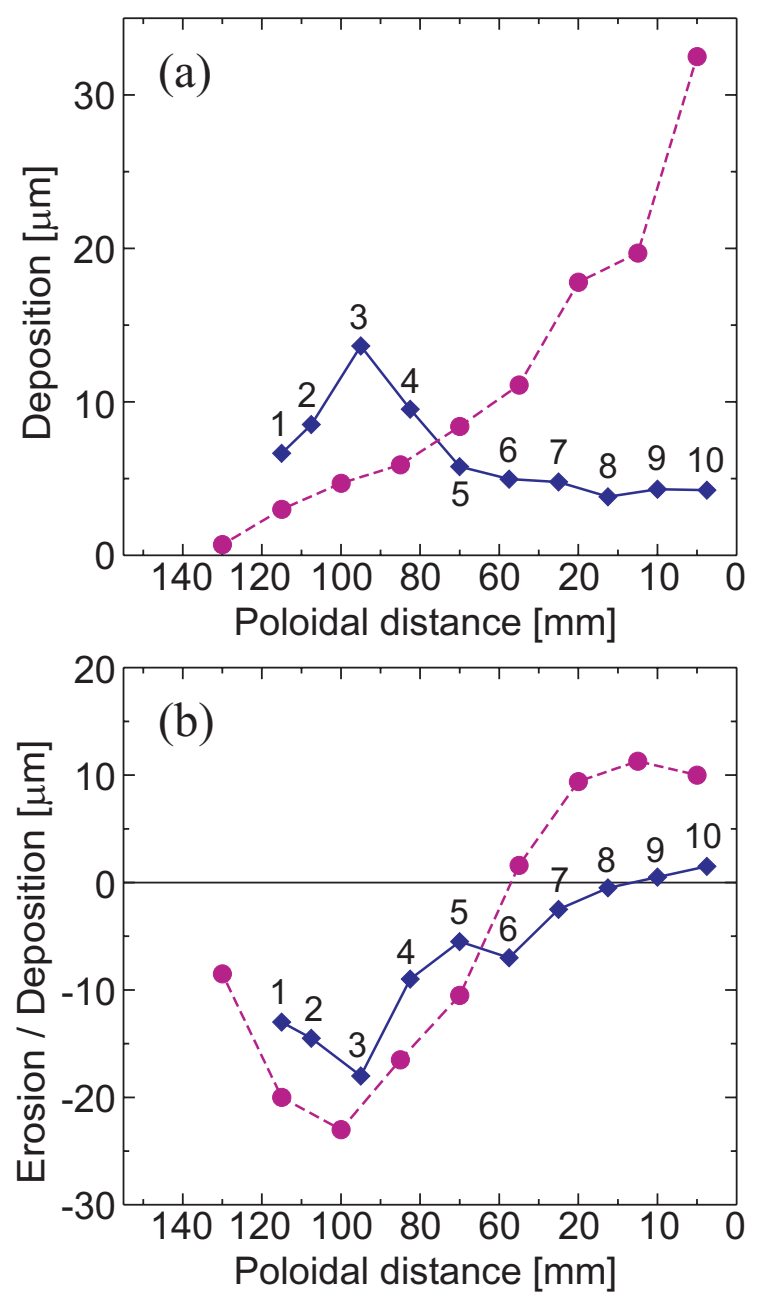

Fig. 2. (a): Thickness of the deposited layer in the marker holes of the central column of the original tile (diamonds) and the refined tile (circles). (b): Surface erosion (negative values) and deposition (positive values) near the marker holes of the central column for the original tile (diamonds) and the refined tile (circles). Both graphs are plotted as a function of the poloidal distance from the upper edge of the tile, as indicated in Fig. 1. The drill holes of the original tile are numbered corresponding to Fig. 1. 


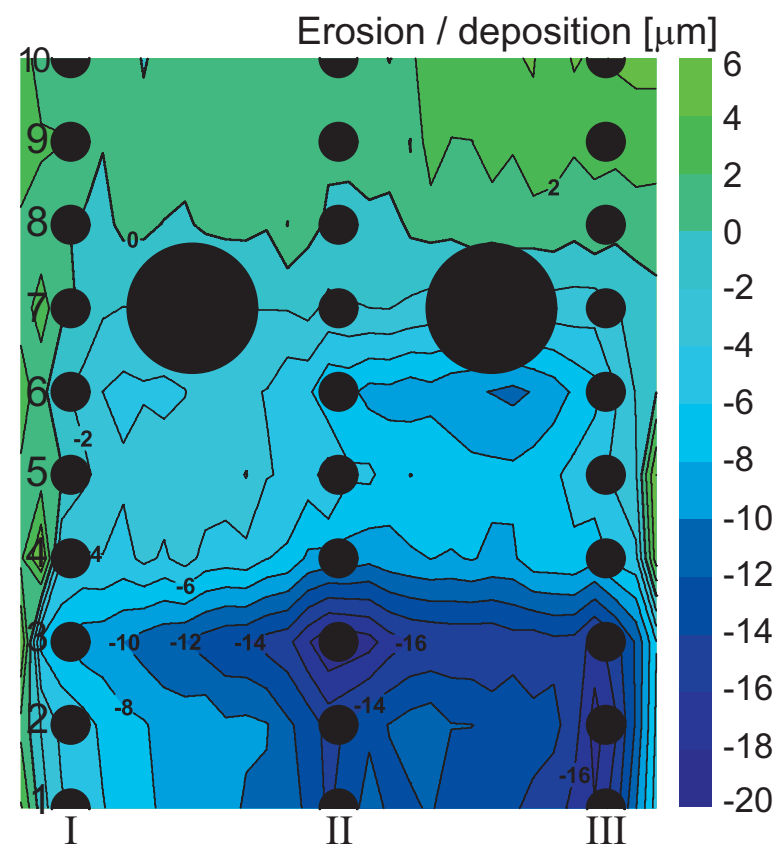

Fig. 3. Two-dimensional reconstruction of the erosion and deposition pattern of the original tile. Negative values represent erosion, positive values deposition. Position of the marker holes and bolt holes are indicated. Note, that the distances between marker hole rows are $12.5 \mathrm{~mm}$ each with exception of the distance between hole rows 1 and 2, which is $7.5 \mathrm{~mm}$. For the equivalent data for the refined tile refer to [7]. 


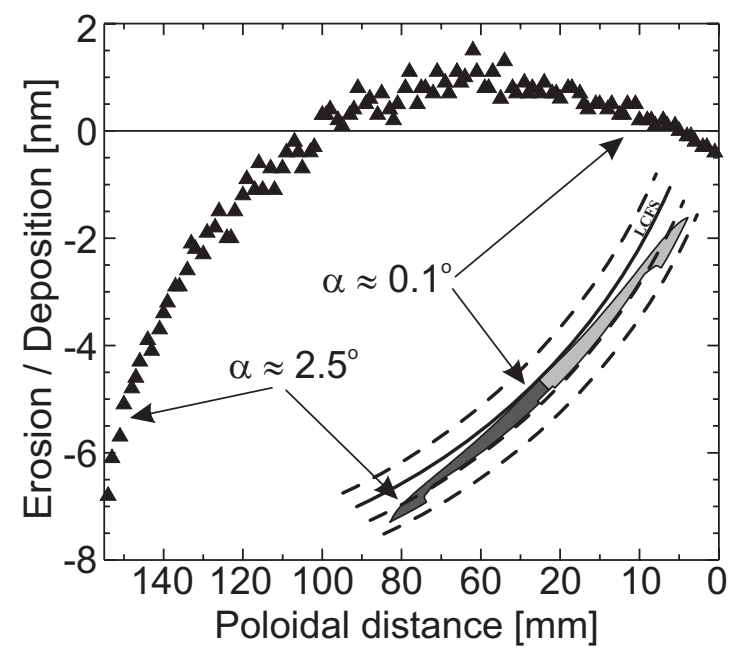

Fig. 4. Erosion and deposition behaviour of the refined tile as a function of the poloidal distance from the upper tile edge as modelled by the ERO code. Poloidal positions with shallow and steeper angles of incidence are indicated. 